\title{
Crystallisation and Initial Characterization of the periplasmic domain of TraG, the Conjugative Entry Exclusion Protein from the F-plasmid
}

\author{
Nicholas Bragagnolo \& Gerald F. Audette \\ Department of Chemistry \& the Centre for Research on Biomolecular Interactions \\ York University, 4700 Keele St., Toronto, ON, M3J 1P3
}

Type IV Secretion Systems (T4SS) are a significant contributor to bacterial evolution and the emergence of antibiotic resistance as they have the unique ability of transmitting mobile DNA elements through bacterial conjugation. The F-plasmid of $E$. coli is representative of conjugative T4SSs found in other clinically relevant gram negative pathogens including Shigella sp., Salmonella sp., Helicobacter pylori etc. TraG is one of the largest proteins expressed from the Fplasmid and plays several roles in the conjugative process. The membrane bound N-terminal domain of TraG is involved in F-pilus synthesis, while the C-terminal periplasmic domain, denoted $\mathrm{TraG}^{*}$, is responsible for entry exclusion (Eex) and mating pair stabilization (Mps). Redundant Fplasmid transfer is prevented by Eex, which occurs when TraG in the donor cell interacts with a cognate TraS in the inner membrane of the recipient cell. Should the TraG-TraS interaction not occur, TraG then interacts with TraN within the outer membrane of donor cell to facilitate Mps; the periplasmic TraG* plays a dynamic role in the Eex/Mps checkpoint of F-mediated conjugative DNA transfer. We have recently crystallized $\mathrm{TraG}^{*}$, and present an initial characterization of the protein. 